\title{
SOME CHARACTERIZATIONS OF HERMITIAN OPERATORS AND RELATED CLASSES OF OPERATORS. I
}

\author{
CHE-KAO FONG AND VASILE I. ISTRĂŢESCU
}

\begin{abstract}
It is shown that, among other things, an operator $T$ is hermitian if and only if $|T|^{2} \leqslant(\operatorname{Re} T)^{2}$. Also, the class of those operators $T$ satisfying $|T|^{2} \geqslant(\operatorname{Re} T)^{2}$ is investigated.
\end{abstract}

0. Introduction. Let $\mathcal{H}$ be a Hilbert space with inner product $\langle$,$\rangle . Let$ $\mathcal{E}(\mathcal{H})$ denote the set of all bounded linear operators on $\mathcal{H}$. For $T \in \mathcal{L}(\mathcal{H})$, the adjoint of $T$ is denoted by $T^{*}$. An operator $T \in \mathcal{L}(\mathcal{F})$ is said to be hermitian if $T=T^{*}$. It is well known that hermitian operators can be characterized in the following way: an operator $T \in \mathcal{L}(\mathcal{H})$ is hermitian if and only if $\langle T x, x\rangle$ is real for all $x$ in $\mathcal{H}$. In what follows we give some characterizations of hermitian operators which involve certain inequalities. By reversing one of these inequalities, we obtain an interesting class of operators which includes hyponormal operators and the class $\theta$ studied in [1] $[4]$.

ACKNOWLEDGEMENT. The first author would like to thank the National Research Council of Canada for financial support as a postdoctoral fellow. This work was done while the second author was a Alexander von Humboldt Fellow at the Johann Wolfgang Goethe University at Frankfurt am Main. He would like to thank Professor Köthe for his warm encouragement, discussions and hospitality. He would also like to thank Professor Campbell for his fruitful correspondence.

1. Some characterizations of hermitian operators. For $T \in \mathcal{L}(\mathcal{H})$, the real part of $T$, denoted by $\operatorname{Re} T$, is defined to be $\left(T+T^{*}\right) / 2$ and the imaginary part of $T$, denoted by $\operatorname{Im} T$, is defined to be $\left(T-T^{*}\right) / 2 i$. It is easy to check that

$$
T=(\operatorname{Re} T)+i(\operatorname{Im} T)
$$

and $T$ is hermitian if and only if $\operatorname{Im} T=0$. We write $|T|$ for the positive square root of $T^{*} T$. Recall that $T \in \mathcal{L}(\mathcal{H})$ is said to be hyponormal if $\left\|T^{*} x\right\| \leqslant\|T x\|$ for all $x$, in other words, $T T^{*} \leqslant T^{*} T$. (See [8].)

LEMMA 1.1. For $T \in \mathcal{L}(\mathcal{H}), T^{*}$ is hyponormal if and only if

$$
|T|^{2} \leqslant(\operatorname{Re} T)^{2}+(\operatorname{Im} T)^{2} .
$$

Received by the editors June 8, 1977 and, in revised form, July 19, 1978 and October 20, 1978. AMS (MOS) subject classifications (1970). Primary 47B15; Secondary 47B20.

Key words and phrases. Hermitian operator, hyponormal operator, real part of an operator, class (WN), spectrum. 
The proof of the above lemma is straightforward and hence omitted. The next theorem shows that a more restrictive inequality forces $T$ to be hermitian.

THEOREM 1.2. If $T \in \mathcal{E}(\mathcal{H})$ and there exists a scalar $\alpha<1$ such that

$$
|T|^{2} \leqslant(\operatorname{Re} T)^{2}+\alpha(\operatorname{Im} T)^{2}
$$

then $T$ is hermitian.

Proof. By Lemma 1.1, $T^{*}$ is hyponormal. In view of [8], to show that $T$ is hermitian, it suffices to show that $\sigma(T)$, the spectrum of $T$, is real. Thus it suffices to show that $\partial \sigma(T)$, the boundary of $\sigma(T)$, is real. Let $\lambda \in \partial \sigma(T)$. Then $\bar{\lambda} \in \partial \sigma\left(T^{*}\right)$. By Problem 63 in [5], $\bar{\lambda}$ is in the approximate point spectrum of $T^{*}$ and hence there exists a sequence $\left\{x_{n}\right\}$ of unit vectors in $\mathcal{H}$ such that $\left\|\left(T^{*}-\bar{\lambda}\right) x_{n}\right\| \rightarrow 0$. Since $T^{*}$ is hyponormal $\left\|(T-\lambda) x_{n}\right\| \rightarrow 0$. Therefore $\left\|T x_{n}\right\| \rightarrow|\lambda|,\left\|(\operatorname{Re} T) x_{n}\right\| \rightarrow|\operatorname{Re} \lambda|$ and $\left\|(\operatorname{Im} T) x_{n}\right\| \rightarrow|\operatorname{Im} \lambda|$. From the assumption we have

$$
\left\|T x_{n}\right\|^{2} \leqslant\left\|(\operatorname{Re} T) x_{n}\right\|^{2}+\alpha\left\|(\operatorname{Im} T) x_{n}\right\|^{2} .
$$

Let $n \rightarrow \infty$, we obtain $|\lambda|^{2} \leqslant|\operatorname{Re} \lambda|^{2}+\alpha|\operatorname{Im} \lambda|^{2}$. Since $\alpha<1, \lambda$ must be real. The proof is complete.

An immediate consequence of the above theorem is the following characterization of hermitian operators:

THEOREM 1.3. An operator $T \in \mathcal{L}(\mathcal{H})$ is hermitian if and only if the inequality $|T|^{2} \leqslant(\operatorname{Re} T)^{2}$ holds.

The following corollary gives a characterization of real scalars.

COROllary 1.4. An operator $T$ satisfies the following condition (C) if and only if $T$ is a real scalar:

(C) $(T-\lambda) x \perp(T-\bar{\lambda}) x \Rightarrow \lambda$ is real or $x=0$.

Proof. Note that if $\|x\|=1$, then

$$
\langle(T-\lambda) x,(T-\bar{\lambda}) x\rangle=\lambda^{2}-2 \lambda\langle(\operatorname{Re} T) x, x\rangle+\left\langle|T|^{2} x, x\right\rangle .
$$

Hence condition (C) means that, for $\|x\|=1$, the quadratic equation (with $\lambda$ as an unknown)

$$
\lambda^{2}-2 \lambda\langle(\operatorname{Re} T) x, x\rangle+\left\langle|T|^{2} x, x\right\rangle=0
$$

has only real solutions. Therefore its discriminant, namely $4\left\{\langle(\operatorname{Re} T) x, x\rangle^{2}-\right.$ $\left.\left\langle|T|^{2} x, x\right\rangle\right\}$, must be nonnegative. Hence, by the Schwarz inequality,

$$
\left\langle|T|^{2} x, x\right\rangle \leqslant\langle(\operatorname{Re} T) x, x\rangle^{2} \leqslant\left\langle(\operatorname{Re} T)^{2} x, x\right\rangle .
$$

Since $x$ is an arbitrary unit vector, we must have $|T|^{2} \leqslant(\operatorname{Re} T)^{2}$. By Theorem 1.3, $T$ is hermitian.

Fix some unit vector $x$. Let $\alpha=\langle T x, x\rangle, \beta=\left(\|T x\|^{2}-\alpha^{2}\right)^{1 / 2}$ and $\lambda=\alpha$ $+i \beta$. Then one can check that $(T-\lambda) x \perp(T-\bar{\lambda}) x$. By condition (C), we 
have $\beta=0$, that is $\left\langle T^{2} x, x\right\rangle=\alpha^{2}$. We have shown that $\left\langle T^{2} x, x\right\rangle=\langle T x, x\rangle^{2}$ for all $x$ with $\|x\|=1$. Now it is easy to see that $T$ must be a scalar.

LEMMA 1.5. An operator $T$ is hyponormal if and only if

$$
|T|^{2} \geqslant(\operatorname{Re} T)^{2}+(\operatorname{Im} T)^{2}
$$

Again, a more restrictive inequality implies that $T$ is hermitian.

THEOREM 1.6. An operator $T$ is hermitian if and only if there exists $\alpha>1$ such that

$$
|T|^{2} \geqslant(\operatorname{Re} T)^{2}+\alpha(\operatorname{Im} T)^{2} .
$$

The proof of the above theorem can be done in the same way as that of Theorem 1.2 and hence is omitted.

Theorem 1.2 can be proved in a different way without using the result of Putnam [8] as follows.

AN ALTERNATIVE PROOF OF THEOREM 1.2. For convenience, write $A=\operatorname{Re} T$ and $B=\operatorname{Im} T$. Then it is easy to check that $|T|^{2} \leqslant A^{2}+\alpha B^{2}$ is equivalent to

$$
(1-\alpha) B^{2} \leqslant i(B A-A B) \text {. }
$$

We claim that (*) leads to $B=0$. Suppose that, on the contrary, $B \neq 0$. Then there is a spectral projection $E$ of $B$ such that $(1-\alpha) B^{2} E \geqslant \varepsilon E$ for some $\varepsilon>0$. From (*) we have

$$
\begin{aligned}
\varepsilon E & \leqslant(1-\alpha) B^{2} E=(1-\alpha) E B^{2} E \\
& \leqslant i E(B A-A B) E=i((B E)(E A E)-(E A E)(B E)) .
\end{aligned}
$$

(Note that $B E=E B=E B E$.) Let $A_{0}$ and $B_{0}$ be the compressions of $A$ and $B$ to $E \mathcal{H}$ respectively. Then we have $\varepsilon \leqslant i\left(B_{0} A_{0}-A_{0} B_{0}\right)$. It is well known that this is impossible. (An easy way to see this is the following. Let $\beta>0$ be large enough such that $C=B_{0}+\beta \geqslant 0$ and let $S=A_{0} C$. Then $\sigma(S)=$ $\sigma\left(C^{1 / 2} A_{0} C^{1 / 2}\right)$ is real. On the other hand, $2(\operatorname{Im} S)=i\left(B_{0} A_{0}-A_{0} B_{0}\right) \geqslant \varepsilon$. Hence if $\lambda \in W(S)$, the numerical range of $S$, then $2(\operatorname{Im} \lambda) \geqslant \varepsilon>0$. This contradicts the fact that $\sigma(S) \subseteq W(S)^{-}$.)

Based on the above proof of Theorem 1.2, we have the following result.

THEOREM 1.7. If $T$ is a hyponormal operator such that $\operatorname{Re} T$ has an eigenvalue, then $T$ has a normal direct summand. If, furthermore, the eigenvectors of $\operatorname{Re} T$ span the whole space $\mathcal{H}$, then $T$ is normal.

Proof. Let $A=\operatorname{Re} T$ and $B=\operatorname{Im} T$. Since $T$ is hyponormal, it is easy to check that $i(A B-B A) \geqslant 0$. Let $\lambda$ be an eigenvalue of $A$ and $M=\{x \in \mathcal{H}$ : $A x=\lambda x\}$. Let

$$
\left(\begin{array}{ll}
\lambda & 0 \\
0 & A_{2}
\end{array}\right) \text { and }\left(\begin{array}{ll}
B_{1} & C \\
C^{*} & B_{2}
\end{array}\right)
$$

be the matrix representations of $A$ and $B$ respectively according to the decomposition $\mathcal{H}=M \oplus M^{\perp}$. Then 


$$
0 \leqslant i(A B-B A)=\left(\begin{array}{cc}
0 & i C\left(\lambda-A_{2}\right) \\
-i\left(\lambda-A_{2}\right) C^{*} & i\left(A_{2} B_{2}-B_{2} A_{2}\right)
\end{array}\right) .
$$

Hence $C\left(\lambda-A_{2}\right)=0$. Since $\lambda-A_{2}$ is one-one, we have $C=0$. Hence $M$ reduces $A$ and $B$ and $T \mid M=\lambda+i B_{1}$ is normal. In case the eigenvectors of $A$ span $\mathcal{H}, A$ is diagonal and the above argument shows that $A B=B A$.

2. A class of operators. For convenience, we denote by (WN) the class of all operators $T$ satisfying the inequality $|T|^{2} \geqslant(\operatorname{Re} T)^{2}$. (The letters WN suggest "weakly normal"). By Lemma 1.5, every hyponormal operator is in (WN). By [4], $T$ is in (WN) if $T^{*}+T$ and $T^{*} T$ commutes.

Operators in (WN) are not necessarily normal, even if the underlying Hilbert space is finite dimensional. For example, if $T=\left(\begin{array}{cc}i & 1 \\ 0 & -i\end{array}\right)$, then

$$
|T|^{2}-(\operatorname{Re} T)^{2}=\left(\begin{array}{cc}
3 / 4 & -i \\
i & 7 / 4
\end{array}\right) \geqslant 0 \text {. }
$$

A reasonable conjecture is the following:

(S) If $T \in \mathcal{L}(\mathcal{H})$ is in (WN) and the spectrum $\sigma(T)$ is real, then $T$ is hermitian.

It is known that, if, in addition, $T$ is hyponormal or $T+T^{*}$ commutes with $T^{*} T$, then the conjecture holds. We shall see that the conjecture holds in the finite dimensional case.

Next we list some spectral properties of operators in (WN).

Proposition 2.1. Suppose that $T \in \mathcal{L}(\mathcal{H})$ is in $(W N)$. Then we have

(i) if $\lambda$ is a real number, then $T-\lambda$ is also in $(W N)$.

(ii) $\left\|(T-\lambda)^{*} x\right\| \leqslant 3\|(T-\lambda) x\|$ for all $x$ in $\mathcal{H}$ and all real numbers $\lambda$.

(iii) If $M$ is an invariant subspace of $T$, then $T \mid M$ is also in (WN); if, furthemore, $T \mid M$ is hermitian, then $M$ reduces $T$.

(iv) If $\lambda$ is a real eigenvalue of $T$, then the eigenspace $\operatorname{ker}(T-\lambda)$ reduces $T$.

(v) If $\lambda$ is real and $(T-\lambda)^{n} x=0$ for some $n \geqslant 1$, then $(T-\lambda) x=0$.

Proof. (i) is straightforward to check.

(ii) By (i), it suffices to consider the case when $\lambda=0$. Now

$$
\begin{aligned}
\left(\left\|T^{*} x\right\|-\|T x\|\right)^{2} & \leqslant\left\|\left(T+T^{*}\right) x\right\|^{2} \\
& =4\left\langle(\operatorname{Re} T)^{2} x, x\right\rangle \leqslant 4\left\langle|T|^{2} x, x\right\rangle=(2\|T x\|)^{2} .
\end{aligned}
$$

Hence $\left\|T^{*} x\right\| \leqslant 3\|T x\|$.

(iii) Let $S=T \mid M$ and $P$ be the orthogonal projection onto $M$. Let $\left(\begin{array}{ll}S & A \\ 0 & B\end{array}\right)$ be the matrix representation of $T$ with respect to the decomposition $\mathcal{H}=M \oplus$ $M^{\perp}$. Then

$$
\begin{aligned}
|S|^{2} & =P|T|^{2} P\left|M \geqslant P(\operatorname{Re} T)^{2} P\right| M \\
& =(\operatorname{Re} S)^{2}+\left(A A^{*}\right) / 4 \geqslant(\operatorname{Re} S)^{2} .
\end{aligned}
$$

In case $S$ is hermitian, $|S|^{2}=(\operatorname{Re} S)^{2}$ and hence $A=0$.

(iv) follows from (iii) and (v) follows from (iv). 
COROLlary 2.2. If $T$ is an operator defined on a finite dimensional space $\mathcal{H}$, $\sigma(T)$ is real and $|T|^{2} \geqslant(\operatorname{Re} T)^{2}$, then $T$ is hermitian.

Other partial results related to conjecture (S) are stated in the following two theorems.

THEOREM 2.3. If $T \in \mathcal{L}(\mathcal{H})$ is in $(W N)$ and $T$ is similar to a hermitian operator, then $T$ is hermitian.

THEOREM 2.4. If $T \in \mathcal{L}(\mathcal{H})$ is in $(W N), \sigma(T)$ is real and there is a polynomial $p$ such that $p(T)=H$ is hermitian, then $T$ is hermitian.

Theorem 2.3 follows from (ii) in Proposition 2.1 and Theorem 4 in [9]. Now we sketch the proof of Theorem 2.4 which makes use of direct integral theory. Let $\mathcal{Z}$ be the abelian von Neumann algebra generated by $H$. Then the underlying Hilbert space $\mathcal{H}$ can be expressed as a direct integral

$$
\mathscr{H}=\int^{\oplus} \mathcal{H}(\xi) d \mu(\xi)
$$

such that each operator in $\mathcal{Z}$ is diagonal and each operator in $\mathcal{Z}^{\prime}$ the commutant of $\mathscr{Z}$, is decomposable. In particular $H=\int^{\oplus} h(\xi) d \mu(\xi)$ for some $h \in L^{\infty}(\mu)$ and since $T \in \mathscr{Z}^{\prime}$ we have

$$
T=\int^{\oplus} T(\xi) d \mu(\xi)
$$

Since $T$ is in (WN), it is easy to show that $T(\xi)$ is in (WN) for almost all $\xi$. Also, since $\sigma(T)$ is real, $\sigma(T(\xi))$ is real for almost all $\xi$. From $p(T)=H$, we have $p(T(\xi))=h(\xi)$ for almost all $\xi$. By (iv) and (v) in Proposition 2.1, it is not hard to see that $T(\xi)$ is hermitian for almost all $\xi$. Therefore $T$ is hermitian.

The next result is due to Professor S. L. Campbell who has kindly allowed us to reproduce it here. It states that, for any operator $T, T-\lambda$ is in (WN) if $|\operatorname{Im} \lambda|$ is large enough.

THEOREM 2.5. Given any $T \in \mathcal{L}(\mathcal{H})$, there exists $\alpha>0$ such that $T-\lambda$ is in $(W N)$ for all $\lambda$ satisfying $|\operatorname{Im} \lambda| \geqslant \alpha$.

Proof. By direct computation, we have

$$
|T-\lambda|^{2}-(\operatorname{Re}(T-\lambda))^{2}=|T|^{2}-(\operatorname{Re} T)^{2}+|\operatorname{Im} \lambda|^{2}-i(\operatorname{Im} \lambda)\left(T-T^{*}\right) .
$$

Hence $(\operatorname{Im} \lambda)^{-2}\left(|T-\lambda|^{2}-|\operatorname{Re}(T-\lambda)|^{2}\right) \rightarrow 1$ as $|\operatorname{Im} \lambda| \rightarrow \infty$. The proof is complete.

\section{REFERENCES}

1. S. L. Campbell, Linear operators for which $T^{*} T$ and $T+T^{*}$ commute, Pacific J. Math. 61 (1975), 53-57.

2. , Operator-valued inner functions analytic on the closed unit disc. II, Pacific J. Math. 60 (1975), 37-50. 
3. S. L. Campbell and R. Gellar, Spectral properties of linear operators which $T^{*} T$ and $T+T^{*}$ commute, Proc. Amer. Math. Soc. 60 (1976), 197-202.

4. _L Linear operators for which $T^{*} T$ and $T+T^{*}$ commute. II, Trans. Amer. Math. Soc. 226 (1977), 305-319.

5. P. R. Halmos, A Hilbert space problem book, Van Nostrand, Princeton, N.J., 1967.

6. V. I. Istrătescu, On some hyponormal operators, Pacific J. Math. 22 (1967), 413-417.

7. __ Topics in linear operator theory, Accademia Nazionale dei Lincei, Roma, 1978.

8. C. R. Putnam, On the spectra of semi-normal operators, Trans. Amer. Math. Soc. 119 (1965), 509-523.

9. M. Radjabalipour, On normality of operators, Indiana Univ. Math. J. 23 (1974), 623-630.

10. A. E. Taylor, Theorems on ascent, descent, nullity and defect of linear operators, Math. Ann. 163 (1966), 18-49.

Department of Mathematics and Statistics, Untversity of Guelph, Guelph, Ontario, NIG 2W1, CANADA (Current address of C. K. Fong)

Fachbereich Mathematik, Johann Wolfgang Goethe Universität, Robert Mayerstrasse 6-10, 600 Frankfurt am Main 1, Federal Republic of Germany

Current address (V. I. Istrătescu): Fachbereich Mathematik, Universität Konstanz, Universitätstrasse 10, Konstanz, Federal Republic of Germany 